\section{Guideline zur Abklärung des palpablen Mammabefundes}

\author{
Arbeitsgruppe "Guidelines Mammadiagnostik»"
}

\section{Präambel}

Im weiten Bereich der Qualitätssicherung im Gesundheitswesen stellen Guidelines ein wichtiges und sinnvolles Segment dar, welches der Ärztin und dem Arzt in komplexen und sich rasch verändernden Fragestellungen in Diagnose, Therapie und medizinischer Technik eine wertvolle Hilfeleistung bietet. Guidelines sollen sich auf Evidenz basierten Publikationen und Expertenmeinungen abstützen und stellen damit den aktuellen Stand des Wissens dar. Sie gelten für den Regelfall; sie sind somit keine in jedem Einzelfall gültige Handlungsanweisungen und damit auch keine Untersuchungs- oder Behandlungsalgorithmen. Sie lassen der Ärztin und dem Arzt die Entscheidung offen, allenfalls von ihnen abzuweichen. Die individuelle Situation der Patientin, des Patienten - insbesondere bei Polymorbidität - ist zu berücksichtigen und zwingt unter Umständen dazu, von den Vorgaben einer Guideline abzuweichen. Ein solches Vorgehen kann und sollte begründet werden. Sofern eine Guideline gewisse qualitative Kriterien und Anforderungen erfüllt, ist sie ein geeignetes Mittel, den medizinischen Entscheidungsprozess zu erleichtern und damit auch die Qualität der ärztlichen Versorgung zu verbessern, auch wenn es äusserst schwierig ist, das Resultat der Anwendung einer Guideline zu evaluieren.

Die Erarbeitung einer Guideline erfordert Zeit, Geduld und viel Engagement einer Expertengruppe. Ist die Guideline nach den Prinzipien der Evidencebased Medicine und eines Expertenkonsensus erstellt,

\footnotetext{
Experten: Prof. Dr. med. J. Benz (SGS), Prof. Dr. med. R. Brun del Re (SGGG). Dr. med. H. H. Brunner (FMH), Dr. med. R. Cranovsky (FMH), Dr. med. E. Eicher (FMH, Koordinator und Leiter der Expertengruppe), Frau Dr. med. E. Garzoli-Meier (SGR), Prof. Dr. med. U. Haller (SGGG), PD Dr. med. J. Hodler (SGR), Prof. Dr. med. U. Laffer (SGC), Dr. med. C. Moll (SGKZ), Dr. med. R. Schibli (SGIM), Dr. med. M. Trutmann (Redaktor SÄZ), Dr. med. H.-R. Zenklusen (SGP), Frau Dr. med. F. Zogg-Harnischberg (SGAM);

Beteiligte Fachgesellschaften: Schweizerische Gesellschaft für Allgemeinmedizin (SGAM), Schweizerische Gesellschaft für Chirurgie (SGC), Schweizerische Gesellschaft für Gynäkologie und Geburtshilfe (SGGG), Schweizerische Gesellschaft für Innere Medizin (SGIM), Schweizerische Gesellschaft für Radiologie (SGR), Schweizerische Gesellschaft für Pathologie (SGP), Schweizerische Gesellschaft für Plastisch-Rekonstruktive und Ästhetische Chirurgie, Schweizerische Gesellschaft für Senologie (SGS), Schweizerische Gesellschaft für Klinische Zytologie (SGKZ);

Koordination: FMH
}

so müsste sie eigentlich weltweit immer identisch sein. Gesundheitssysteme, Ausbildung der Ärztinnen und Ärzte, Behandlungstraditionen, ethische und religiöse sowie epidemiologische Unterschiede können jedoch eine Guideline beeinflussen, so dass sie nicht tel quel von einem anderen Land übernommen werden kann.

Im vorliegenden Fall hat die Arbeitsgruppe die Guideline der Canadian Medical Association [1] als Arbeitsgrundlage übernommen.

\section{Relevanz}

Das Mammakarzinom ist bei der Frau das am häufigsten vorkommende Karzinom. Der palpable Befund in der Mamma ist für die Praxis hoch relevant, genaue statistische Zahlen über dessen Vorkommen sind allerdings nicht verfügbar. Die Mortalität von Patientinnen mit Mammakarzinom betrug 1996 29,2 pro 100000 Personen [2].

\section{Ziel der vorliegenden diagnostischen Guideline}

Die Vereinheitlichung der Untersuchungsmethoden ist anzustreben. Damit kann die Verunsicherung der Patientinnen abgebaut werden. Noch nicht generell verbreitete Untersuchungsmethoden sind in dieser Guideline nicht berücksichtigt.

\section{Methodik}

Die Verbindung der Schweizer Ärztinnen und Ärzte FMH hat in der Erkenntnis, dass aus den schon genannten Gründen Handlungsbedarf besteht, sämtliche involvierten Fachgesellschaften angeschrieben und gebeten, Experten in eine Arbeitsgruppe zu delegieren. Diese wurde durch die FMH koordiniert und geleitet. Die Experten haben sich während 2 Jahren regelmässig getroffen, die Literatur computerunterstützt gesucht mit MEDLINE ab 1966 und CANCERLIT ab 1985 bis 1999; die relevante Literatur, insbesondere kürzlich publizierte Guidelines [1, 3], wurde durchgearbeitet und auf ihre Evidenz geprüft. Die Evidenzgrade der einzelnen Aussagen der Kurzfassung sind in den Begründungen der Empfehlungen mit $\varepsilon_{1}, \varepsilon_{2}, \varepsilon_{3}$ und $\varepsilon_{4}$ gekennzeichnet. Evidenzgrad V $\left(\varepsilon_{5}\right)$ entspricht dem Konsens der Arbeitsgruppe und wird nicht speziell vermerkt. Nach Fertigstellung der Guideline (6. Fassung) wurde sie an alle beteiligten Fachgesellschaften gesandt und durch je 10 Experten überprüft. Die Vernehmlassungsresultate wurden daraufhin erneut in der Expertengruppe diskutiert und in eine neue definitive Fassung gebracht. Eine klinische Testung wurde während 3 Monaten unter 60 Mitgliedern der verschiedenen involvierten Fachgesellschaften als letzter Schritt vor der definitiven Genehmigung durch die Experten ausgeführt.

\section{Aktualisierung/Ausserkraftsetzung}

Die Guideline hat ab Veröffentlichung eine vorläufige Gültigkeit von 2 Jahren, muss aber bei Bedarf bereits vorher überarbeitet werden. Verantwortlich ist die Arbeitsgruppe unter Koordination der FMH. 
Grad der Evidenz (Canadian Task Force on the Periodic Health Examination) [1].

Grad I: Die Evidenz ist aufgrund randomisierter kontrollierter Studien (oder Metaanalysen) von genügendem Umfang derart, dass die Gefahr, dass sie falsch positive oder falsch negative Resultate beinhalten, gering ist.

Grad II: Die Evidenz basiert auf randomisierten kontrollierten Studien, welche jedoch zu klein sind, um ihnen Grad I zuzusprechen; sie können positive Trends, welche jedoch statistisch nicht signifikant sind, oder gar keine Trends zeigen. Sie sind mit einem hohen Risiko falsch negativer Resultate verbunden.

Grad III: Die Evidenz basiert auf nicht randomisierten Kontroll- oder Kohortenstudien, Fallserien, Fallkontrollstudien oder Querschnittsstudien.

Grad IV: Die Evidenz basiert auf der Meinung angesehener Experten oder Expertengremien, wie sie in publizierten Konsensuskonferenzen oder in Guidelines angegeben werden.

\section{Kurzfassung}

Ein palpabler Befund in der Mamma ist häufig. Obwohl es sich dabei meistens um gutartige Veränderungen handelt, muss trotzdem auch immer an die Möglichkeit eines Karzinoms gedacht werden.

Diese "Guideline» soll Ärztinnen und Ärzten Informationen und Empfehlungen zur weiteren $\mathrm{Ab}$ klärung des palpablen Knotens liefern, um zielgerichtet und rasch ein Karzinom zu bestätigen oder auszuschliessen.

Die Faktoren, welche die notwendigen Entscheidungen beeinflussen, wurden kritisch geprüft. Es wird bei jeder Empfehlung auf Begründung und Evidenz eingegangen. Auf das Vorgehen bei einem zufällig mammographisch entdeckten Befund wird nicht eingegangen.

1. Die Abklärung eines palpablen Befundes in der Mamma erfolgt durch Anamnese, klinische Untersuchung und weitere Untersuchungen.

2. Mit der Anamnese ist zu klären, wann der tastbare Befund erstmals bemerkt wurde und ob er sich seither verändert hat, ob bereits früher Abklärungen durchgeführt oder ein Mammakarzinom behandelt wurden. Risikofaktoren sollen erfragt werden. Deren Fehlen darf aber keinen Einfluss auf die weitere Abklärung des palpablen Befundes haben.

3. Mit der klinischen Untersuchung wird nach Merkmalen gesucht, die zur Unterscheidung zwischen benignen und malignen Befunden beitragen.

4. Die Mammographie kann bei palpablen Befunden diagnostisch weiterhelfen und ausserdem nicht palpable Befunde, auch auf der Gegenseite, aufdecken.
Grad V: Die Evidenz basiert auf der Meinung derjenigen Personen, welche diese Guidelines geschrieben oder aktualisiert haben, beruhend auf ihrer Erfahrung, ihrer Kenntnis der einschlägigen Literatur und der Diskussion mit ihren Fachkollegen.

\section{Dissemination und Implementierung}

Die Guideline steht auch im Internet zur Verfügung (www.emh.ch) und wird durch die Fachgesellschaften verbreitet. Die Guideline soll für Aus-, Weiter- und Fortbildungszwecke verwendet werden; weitere gedruckte Exemplare können bei der FMH bezogen werden.

\section{Finanzierung}

Bei der Erstellung der Guideline wurden keine Sponsorengelder eingesetzt, die Finanzierung der Expertengruppe, die Sekretariatsarbeiten, die Vervielfältigung der Literatur, erfolgte durch die FMH. Die jeweiligen Arbeitsfassungen wurden durch den Chefredaktor der Schweizerischen Ärztezeitung vorgenommen.

5. Die Ultraschalluntersuchung ermöglicht eine Differenzierung zwischen zystischen und soliden Befunden, ergänzt andere bildgebende Verfahren und stellt die Methode der Wahl bei mammographisch dichter Brust, während der Schwangerschaft und in der Stillzeit dar.

6. Die Feinnadelpunktion ermöglicht eine Unterscheidung zwischen zystischen und soliden Befunden und erlaubt es meistens, eine morphologische Diagnose zu stellen. Aspirate sollen immer zytologisch abgeklärt werden.

7. Falls die Dignität eines palpablen Befundes mit den genannten Untersuchungsmethoden nicht feststeht, sollte auf jeden Fall eine histologische Diagnose erzwungen werden.

8. Eine Stanzbiopsie kann oft Malignität bestätigen oder aber ausschliessen und hilft damit, Exzisionen zu vermeiden.

9. Das Exzisat sollte vollständig (weder zerstückelt noch eingeschnitten) entfernt und topographisch markiert werden.

10. Der Einsatz der Magnetresonanz-Tomographie ist nur bei besonderen Fragestellungen indiziert.

11. Die Patientin soll während der gesamten Dauer der Abklärung eingehend informiert werden.

12. Auch wenn kein Hinweis auf ein Karzinom gefunden wurde, ist es empfehlenswert, jährliche Nachkontrollen vorzunehmen. 


\section{Begründung der Empfehlungen}

1. Die Abklärung eines palpablen Befundes in der Mamma erfolgt durch Anamnese, klinische Untersuchung und weitere Untersuchungen.

Obwohl die meisten Palpationsbefunde nicht maligne sind, muss trotzdem ihre Dignität abgeklärt werden. Die ersten Schritte sind Anamnese und klinische Untersuchung. Als weitere Abklärungen folgen: Mammographie, Ultraschall, Feinnadelpunktion und bei Unklarheit Stanzbiopsie oder Exzision. Das Prinzip ist, eine zuverlässige Diagnose mit einem Minimum an diagnostischem Aufwand zu stellen. Bei Frauen mit Brustimplantaten bieten sich als bildgebende Verfahren in erster Linie Ultraschall und ergänzend die Magnetresonanz-Tomographie an. Feinnadelpunktion, Stanzbiopsie oder Exzision dürfen keine Schädigung des Implantates verursachen.

2. Mit der Anamnese ist zu klären, wann der tastbare Befund erstmals bemerkt wurde und ob er sich seither verändert hat, ob bereits früher Abklärungen durchgeführt oder ein Mammakarzinom behandelt wurden. Risikofaktoren sollen erfragt werden. Deren Fehlen darf aber keinen Einfluss auf die weitere Abklärung des palpablen Befundes haben.

Die Wahrscheinlichkeit für das Vorliegen eines Mammakarzinoms ist bei folgenden Faktoren erhöht:

- Zustand nach Biopsie mit atypischer Hyperplasie $\varepsilon_{3}$ [4], lobulärem Carcinoma in situ (LCIS) $\varepsilon_{3}$ [5] oder duktalem Carcinoma in situ (DCIS) $\varepsilon_{3}$ [6];

- Zustand nach invasivem Karzinom $\varepsilon_{3}$ [7];

- Zustand nach Ovarial-, Korpus- oder Kolonkarzinom [8-12];

- Zustand nach thorakaler Bestrahlung wegen Morbus Hodgkin in der Kindheit und Adoleszenz $\varepsilon_{3}[13]$

- positive Familienanamnese (Mutter oder Schwester mit Mammakarzinom) $\varepsilon_{3}$ [14].

Das Risiko für ein Mammakarzinom steigt auch mit zunehmendem Alter $\varepsilon_{3}[3,15]^{2}$. Weiterhin ist das Brustkrebsrisiko bei Frauen mit Nachweis von palpablen Zysten erhöht, und zwar unabhängig von der Art der Zyste $\varepsilon_{1}$ (Metaanalyse, [16]). Es bleibt eine Tatsache, dass die meisten Frauen, die an einem Mammakarzinom erkranken, keine bekannten Risikofaktoren aufweisen und dass bei den meisten Frauen mit bekannten Risikofaktoren nie ein Mammakarzinom auftritt $\varepsilon_{3}[3]$.

3. Mit der klinischen Untersuchung wird nach Merkmalen gesucht, die zur Unterscheidung zwischen benignen und malignen Befunden beitragen.

Zur Untersuchung der Brust gehört auch die Untersuchung der Axilla und der supraklavikulären Region auf verdächtige Lymphknoten. Der günstigste Zeitpunkt für die Untersuchung von Frauen vor der Menopause ist eine Woche nach Beginn der Menstruation $\varepsilon_{4}$ [17]. Eine Beschreibung der Untersuchungstechnik findet sich bei [18] und [19].

Bei der klinischen Untersuchung ist folgenden Punkten besondere Aufmerksamkeit zu schenken:

\section{Inspektion}

Hautveränderungen der Mamillen können durch ein Karzinom verursacht sein (M. Paget) [20]. Der M. Paget gleicht häufig einer Dermatitis, der Befund ist manchmal feucht und ekzematös, manchmal trocken und schuppend und weist gewöhnlich eine Verdickung des Warzenhofs auf. Brennen oder Juckreiz sind mögliche Symptome. Ursache ist eine Infiltration von Karzinom-Zellen aus einem Milchgang in die darüberliegende Haut [18]. Falls mit einer lokalen Therapie keine sofortige Besserung erreicht werden kann, ist eine Biopsie der veränderten Mamille angezeigt. Bei bestätigtem M. Paget ist eine Mammographie durchzuführen.

\section{Palpation}

Glatte, gut begrenzte Befunde sind meist benigne $\varepsilon_{5}$ [1]. Es handelt sich in den meisten Fällen um Zysten oder um Fibroadenome. Weniger glatte und weniger bewegliche Befunde, die schlecht abgrenzbar sind, sind verdächtig auf ein Karzinom. Plattenförmige Indurationen, die in das umgebende Brustdrüsengewebe übergehen, entsprechen meistens mastopathischen Veränderungen. Eine halbmondförmige Verhärtung der unteren Brustfalte bei älteren Frauen kommt durch Kompression des Fettgewebes durch das Gewicht der darüberliegenden Brust zustande. Dieser benigne Befund tritt symmetrisch auf.

\section{Mamillen-Sekretion}

Mamillen-Sekretion ist nicht charakteristisch für ein Karzinom (Tab. 1 und 2). Das Sekret kann serös, blutig, milchig oder purulent sein. Die wichtigsten Risikofaktoren für das Vorliegen eines Karzinoms sind eine einseitige oder eine blutige Sekretion (Cave: okkultes Blut; Nachweis mit Hämostix). Die Abklatschzytologie ist mit 45\% wenig sensitiv; mit dieser Untersuchungsmethode kann deshalb ein Karzinom nicht ausgeschlossen werden.

\section{Schmerzen}

Obwohl Mammakarzinome in der Regel schmerzfrei sind, können sie mit Beschwerden einhergehen $\varepsilon_{3}$ $[18,21]$. Das Vorhandensein oder Fehlen von Schmerz oder Druckempfindlichkeit sollte deshalb die weitere Abklärung eines Palpationsbefundes nicht beeinflussen $\varepsilon_{3}$ [21].

\footnotetext{
${ }^{2}$ In Kanada war die Inzidenz 1992 bei Frauen zwischen 30-39 Jahren 0,35/1000, bei Frauen zwischen 50-59 Jahren 2,2/1000 und bei Frauen zwischen 70-79 Jahren 4,0/1000 [15].
} 


\section{Tabelle 1 (nach [3] und [48])}

Prozentuale Verteilung von Mamillen-Sekretion und Karzinom bei 5305 zytologisch untersuchten Fällen mit Mamillen-Sekretion.

\begin{tabular}{|c|c|c|c|c|c|}
\hline & & $<40$ & $\begin{array}{l}\text { Alter } \\
40-59\end{array}$ & $\geq 60$ & Total \\
\hline \multirow[t]{2}{*}{ serös } & \% Sekretion & 25 & 25 & 1,5 & 52 \\
\hline & \% Karzinome & 0 & 6 & 6 & 12 \\
\hline \multirow[t]{2}{*}{ Milchig } & \% Sekretion & 20 & 11 & - & 31 \\
\hline & \% Karzinome & 3 & 3 & - & 6 \\
\hline \multirow[t]{2}{*}{ Eitrig } & \% Sekretion & 2,5 & 4,5 & 0,1 & 7 \\
\hline & \% Karzinome & 0 & 3 & 6 & 9 \\
\hline \multirow[t]{2}{*}{ Blutig } & \% Sekretion & 3,0 & 6,2 & 2,1 & 11 \\
\hline & \% Karzinome & 13 & 29 & 32 & 74 \\
\hline \multirow[t]{2}{*}{ Total } & \% Sekretion & 51 & 47 & 4 & 100 \\
\hline & \% Karzinome & 16 & 39 & 44 & 100 \\
\hline
\end{tabular}

Tabelle 2 (nach [3] und [48])

Wahrscheinlichkeit in \% nach Alter und Art der Mamillen-Sekretion.

\begin{tabular}{lll}
\hline Sekretion & \multicolumn{2}{c}{ Alter (Jahre) } \\
& $<60$ & $\geq 60$ \\
\hline Nicht blutig & $<1 \%$ & $5 \%$ \\
\hline Blutig & $3 \%$ & $9 \%$
\end{tabular}

\section{Gutartige Mammabefunde}

Bei harmlosen Befunden handelt es sich meistens um Fibroadenome, fibrozystische Veränderungen und Zysten.

\section{Fibroadenome}

Der Palpationsbefund bei Fibroadenomen ist glatt, gut abgegrenzt, derb und gut verschieblich.

\section{Fibrozystische Veränderungen}

Das häufigste Symptom sind Schmerzen, welche zyklusabhängig auftreten: Sie nehmen nach der Ovulation bis zum Menstruationsbeginn zu und verschwinden dann rasch während der Menstruation. Sie können wenige Tage, aber auch 2-3 Wochen lang dauern. Sie strahlen oft in die Schultern und Arme aus. Fibrozystische Veränderungen sind in der Regel symmetrisch und finden sich am häufigsten in den oberen, äusseren Quadranten. Das Gewebe ist elastisch oder kann plattenförmig induriert sein. Diese Veränderungen gehen typischerweise ohne klare $\mathrm{Ab}$ grenzung in das umgebende normale Drüsengewebe über. Im Gegensatz dazu sind Befunde, ob maligne oder benigne, deutlicher abgrenzbar.

\section{Zysten}

Zysten sind eher rund und können schmerzhaft oder druckempfindlich sein. Liegen sie oberflächlich, sind sie beweglich und gut umschrieben; liegen sie tiefer, ist ihre Beweglichkeit eingeschränkt und die Abgrenzung zur Umgebung schwierig. Sie können eher weich sein, sind aber meist recht hart, wenn sie prall- gefüllt sind. In der Ultraschalluntersuchung kann ihre Komprimierbarkeit nachgewiesen werden.

\section{Klinische Beurteilung}

Die klinische Beurteilung hängt im wesentlichen vom Wissen und von der Erfahrung des Untersuchers ab. Die Technik der Brustuntersuchung muss erlernt werden und wird mit zunehmender Erfahrung besser $\varepsilon_{3}[22]^{3}$.

Jeder palpable Befund muss weiter abgeklärt werden. Die Reihenfolge der nachfolgenden Abklärungsschritte (Mammographie, Ultraschall, Feinnadelpunktion) hängt vom Alter der Frau, der Art des Palpationsbefundes und der lokal verfügbaren Technologie $a b$.

4. Die Mammographie kann bei palpablen Befunden diagnostisch weiterhelfen und ausserdem nicht palpable Befunde, auch auf der Gegenseite, aufdecken.

Bei mammographisch dichtem Brustdrüsengewebe ist die Aussagekraft der Mammographie eingeschränkt. Dies trifft oft $\mathrm{zu}$ bei jungen Frauen und/oder bei Frauen, welche mit Hormonsubstitution behandelt werden $[3,18]$. Ab dem 30. Lebensalter jedoch steigt der Wert der Untersuchung, weshalb die Mammographie von diesem Zeitpunkt an zunehmend häufiger zur weiteren Abklärung eines verdächtigen Palpationsbefundes einbezogen werden soll $\varepsilon_{4}$ [18]. Unregelmässige und gruppierte Mikroverkalkungen im Bereich der Resistenz erhöhen den Karzinomverdacht. Die Mammographie kann oft diagnostisch weiterhelfen und liefert ausserdem Informationen über die gesamte Brustdrüse, auch auf der Gegenseite.

Die Aufnahmen müssen auf jeder Seite in zwei Ebenen angefertigt werden, allenfalls mit Vergrösserung der abnormen Befunde [25]. Aufgrund einer verdächtigen Mammographie ist die Wahrscheinlichkeit für das Vorliegen eines Karzinoms erhöht, aber eine unauffällige Mammographie kann bei klinischem Verdacht ein Karzinom nicht ausschliessen. Deshalb soll bei klinischem Verdacht und unauffälliger Mammographie weiter abgeklärt werden.

Ob die Mammographie oder die Feinnadelpunktion zuerst durchgeführt wird, hängt vom Untersucher ab. Ärzte, die mit der Feinnadelpunktion Erfahrung haben, führen diese meist direkt durch und können damit rascher eine sichere Diagnose erhalten. Die Feinnadelpunktion führt in seltenen Fällen zu Artefakten in der Mammographie. Die Mammographie kann deshalb entweder vorgezogen oder erst nach einer Wartezeit von 14 Tagen durchgeführt werden $\varepsilon_{4}$ [26].

${ }^{3}$ In einer Studie von Rimsten et al. [23] wurde die Diagnose Karzinom bei 92,5\% der klinisch stark verdächtigen Palpationsbefunde bestätigt, wenn der Untersucher erfahren war. Van Dam et al. fanden, dass die klinische Untersuchung einen positiv prädiktiven Wert von 73\% und einen negativ prädiktiven Wert von $87 \%$ hatte [24]. 
5. Die Ultraschalluntersuchung ermöglicht eine Differenzierung zwischen zystischen und soliden Befunden, ergänzt andere bildgebende Verfahren und stellt die Methode der Wahl bei mammographisch dichter Brust, während der Schwangerschaft und in der Stillzeit dar.

Ein erfahrener Untersucher kann mit Ultraschall zuverlässig eine Zyste erkennen $\varepsilon_{3 / 4}$ [27].

Bei der Differenzierung solider Raumforderungen hingegen nimmt die Sonographie einen geringeren Stellenwert ein, nicht zuletzt aufgrund der z.T. deutlichen Variabilität zwischen verschiedenen Untersuchern und sogar bei wiederholt durchgeführten Sonographien durch den gleichen Untersucher $\varepsilon_{3}$ [28]. Sonographische Befunde haben bei jüngeren einen höheren Stellenwert als bei älteren Frauen und können die Mammographie bei Frauen unter 40 Jahren sogar hinsichtlich richtig positiver Malignitätsdiagnosen übertreffen $\varepsilon_{4}$ [29]. Sonographische Zeichen der Malignität sind unscharfe Begrenzung, dorsaler Schallschatten, inhomogene Struktur $\varepsilon_{4}$ [30] sowie Unterbrechung anatomischer Grenzflächen.

Die Sonographie ist geeignet zur gezielten Biopsie vor allem beim Vorliegen von multiplen palpablen Knoten und bei Verdacht auf Karzinom (Feinnadelpunktion oder histologische Untersuchung) $\varepsilon_{3}$ [31].

\section{Die Feinnadelpunktion ermöglicht eine Unter- scheidung zwischen zystischen und soliden Be- funden und erlaubt es meistens, eine morpholo- gische Diagnose zu stellen. Aspirate sollen immer zytologisch abgeklärt werden.}

Die Feinnadelpunktion ist kostengünstig. Nach erlernter Technik ist diese Methode leicht auszuführen und bedarf keiner weiteren Vorbereitungen. Die Feinnadelpunktion ist praktisch schmerzfrei und kann gewöhnlich ohne Lokalanästhesie durchgeführt werden [32].

\footnotetext{
${ }^{4}$ Die Diagnose kann aufgrund der Feinnadelpunktion in bis zu 95\% der Fälle richtig gestellt werden [34]. In einer Verlaufsstudie mit insgesamt 835 Palpationsbefunden war die Feinnadelpunktion $135 \mathrm{mal}$ positiv mit nur einer falsch positiven Beurteilung $(0,7 \%)$, 92mal negativ mit 14 falsch negativen Befunden $(15,2 \%) \varepsilon_{3}$ [35]. Kürzlich wurde in einer Vergleichsstudie zwischen Feinnadelpunktion und Stanzbiopsie bei palpablen Mammatumoren eine Sensitivität von 97,5\% für die Feinnadelpunktion und eine von 90\% für die Stanzbiopsie gefunden [36]. Die ultraschallgeführte Stanzbiopsie liefert gleichwertige Resultate wie die Feinnadelpunktion [31]

${ }^{5}$ In einer Studie mit 150 Stanzbiopsien bei palpablen Tumoren war die Sensitivität $89 \%$, ansteigend auf $94 \%$ bei Tumoren grösser als 2,5 cm im Durchmesser. Es gab kein falsch positives Resultat [38]. In einer Studie von Donegan war unter 1784 solcher Biopsien (gesammelt in 5 verschiedenen Arbeiten) kein einziges falsch positives Resultat, aber der Anteil der falsch negativen Resultate lag zwischen 1,6\% und 19\% [39].
}

Die Punktion sollte in 90-95\% der Fälle repräsentativ sein [33] $]^{4}$.

Mit der zytologischen Untersuchung eines Feinnadelpunktates kann meistens die morphologische Diagnose sowohl in bezug auf Dignität wie auch Art der Veränderung gestellt werden. Wenn keine malignen Zellen gefunden werden, kann dies den klinischen Eindruck einer gutartigen Veränderung bestätigen. Werden aber maligne Zellen gefunden, erlaubt dies eine bessere Planung des operativen Vorgehens. Ergeben Zytologie, Mammographie und Klinik alle den Verdacht auf ein Karzinom, wird die Diagnose histologisch in über 99\% der Fälle bestätigt. Deuten schliesslich alle 3 Untersuchungen auf eine benigne Veränderung hin, wird in weniger als 0,5\% der Fälle histologisch ein Karzinom gefunden [37].

Die Kombination der klinischen Untersuchung, der Mammographie und der FNP wird als Tripel-Test bezeichnet. Resultate dieses Tests finden sich in Tabelle 3 .

7. Falls die Dignität eines palpablen Befundes mit den genannten Untersuchungsmethoden nicht feststeht, sollte auf jeden Fall eine histologische Diagnose erzwungen werden.

Der Entscheid, unter welchen Bedingungen bei fehlendem positiven zytologischen Befund eine Biopsie durchgeführt werden sollte, stützt sich auf alle verfügbaren Informationen, wie klinische Untersuchung, Mammographie und Ultraschall. Der Kliniker sollte bestrebt sein, Karzinome mit einem Minimum an Biopsien nachzuweisen oder auszuschliessen. Entscheidet man sich für eine Biopsie, kann entweder eine Stanzbiopsie oder eine Exzision durchgeführt werden.

\section{Eine Stanzbiopsie kann oft Malignität bestäti- gen oder aber ausschliessen und hilft damit, Ex- zisionen zu vermeiden.}

Eine Stanzbiopsie wird vor allem als Alternative zur Exzision angewandt. Handgeführte Stanzbiopsien sind genauer, wenn der Palpationsbefund gross ist $\varepsilon_{3}{ }^{5}$. Die dabei entnommenen Gewebezylinder werden histologisch untersucht. Bei einem Karzinom können die Invasion und der Hormonrezeptorstatus bestimmt werden. Nicht repräsentative Proben sind selten.

Kleine Befunde, die nur mit Mühe palpiert werden, können stereotaktisch oder sonographisch gezielt biopsiert werden $\varepsilon_{3}$ [39-41].

Die Diagnose eines Karzinoms sollte möglichst mit Feinnadelpunktion oder Stanzbiopsie und ohne Exzision gestellt werden. Ein zweiter chirurgischer Eingriff kann damit vermieden werden. Dies gestattet eine offene Diskussion mit der Patientin und eine bessere Planung des chirurgischen Verfahrens. 
9. Das Exzisat sollte vollständig (weder zerstückelt noch eingeschnitten) entfernt und mit Angabe der Seitenlokalisation topographisch markiert werden.

Nach einer unvollständigen Exzision, welche den Nachweis eines Karzinoms erbracht hat, muss eine Nachresektion durchgeführt werden, um die Entfernung im Gesunden sicherzustellen. Beim Zweiteingriff ist, insbesondere nach fehlender topographischer Markierung des Exzisates, eine genaue Lokalisierung des Befundes deutlich schwieriger, weil das Exzisionsgebiet durch postoperative Gewebereaktionen verändert ist $\varepsilon_{4}$ [1]. Deshalb sollte beim Ersteingriff eine vollständige Exzision durchgeführt werden. Das Präparat sollte vom Operateur topographisch markiert und nicht eingeschnitten werden. Eine $\mathrm{Ab}$ sicherung kann allenfalls mit einer Schnellschnittuntersuchung erreicht werden.

10. Der Einsatz der Magnetresonanz-Tomographie ist nur bei besonderen Fragestellungen indiziert.

Nach heutigem Wissensstand ist der histologisch und/oder zytologisch nachgewiesene maligne palpable Befund bei mammographisch dringendem Verdacht auf Multizentrizität und/oder Bilateralität mittels Magnetresonanz-Tomographie abzuklären. Bei der Abklärung eines palpablen Befundes bei Implantatträgerinnen kann Magnetresonanz-Tomographie eine wertvolle Zusatzinformation liefern ${ }^{6}$.

\section{Die Patientin soll während der gesamten Dauer der Abklärung eingehend informiert werden.}

Die Entdeckung einer tastbaren Veränderung in der Mamma bereitet der Patientin grosse Angst. Deshalb sollte die Abklärung möglichst ohne Verzug durchgeführt werden. Dies erfordert eine gute Kommunikation und Zusammenarbeit zwischen Hausarzt, Operateur, Radiologe und Pathologe $\varepsilon_{4}$ [1].
Eine gute Kommunikation zwischen Patientin und behandelndem Arzt vermindert nicht nur kurzzeitig die Angst, sondern hat noch viele Monate später einen Einfluss auf das psychische Wohlbefinden $\varepsilon_{3}$ $[46,47]$. Vollständige und mitfühlende Erklärungen bei jedem Abklärungsschritt, genügend Zeit für Fragen und die Ermutigung zur Mitentscheidung sind wichtige Bestandteile der Behandlung.

12. Auch wenn kein Hinweis auf ein Karzinom gefunden wurde, ist es empfehlenswert, jährliche Nachkontrollen vorzunehmen.

Gutartige Befunde in der Mammographie und/oder in der Sonographie und palpable Befunde, die zytologisch nicht maligne sind, sollen jährlich nachkontrolliert werden, um allfällige Veränderungen nicht zu verpassen. Auch wenn feststeht, dass eine tastbare Veränderung nicht maligne ist, kann es ratsam sein, eine oder mehrere Nachkontrollen anzuordnen.

\footnotetext{
${ }^{6}$ Thermographie und "light scanning" sind im Gegensatz dazu obsolete Verfahren. Die Thermographie misst die höhere Wärmeabstrahlung aus einem Mammakarzinom. Sie wurde 1956 von Lawson eingeführt und kann symptomatische Karzinome erkennen [42]. Allerdings konnte gezeigt werden, dass die Sensitivität bei kleinen Tumoren dem Zufall entspricht [43]. Das Beahrs Committee des National Cancer Institute (NCI) empfahl 1977, die Thermographie nicht mehr als Routine-Screening-Methode im Projekt BCDDP (Breast Cancer Detection Demonstration Project) einzusetzen [44]. 1980 kam eine Studie, die 576 Thermogramme aus dem BCDDP von 10 erfahrenen Thermographen blind beurteilen liess, zum Schluss, dass der "Aufdeckungsgrad" («index of detectability") nicht grösser war als der Zufall $\varepsilon_{1}$ [45].
} 


\begin{tabular}{|c|c|c|c|c|c|c|c|c|c|c|c|c|c|c|c|c|c|c|c|}
\hline & \multirow{2}{*}{ 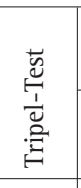 } & \multirow{2}{*}{\multicolumn{2}{|c|}{$\begin{array}{c}\text { if : } \\
z\end{array}$}} & \multirow{2}{*}{\begin{tabular}{|l|}
.. \\
\multirow{0}{0}{} \\
\end{tabular}} & \multirow{2}{*}{\multicolumn{2}{|c|}{ 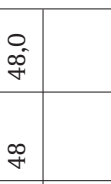 }} & \multirow{2}{*}{ 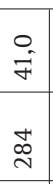 } & \multirow{2}{*}{\multicolumn{2}{|c|}{ 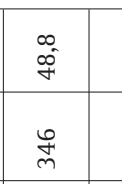 }} & \multirow{2}{*}{$\begin{array}{l}\infty \\
0 \\
- \\
- \\
\hat{n} \\
\end{array}$} & \multirow{2}{*}{$\begin{array}{l}\infty \\
\infty \\
\hat{N}^{-} \\
\hat{2} \\
\end{array}$} & \multirow{2}{*}{$\begin{array}{l}\text { iv } \\
\text { in } \\
\stackrel{n}{m}\end{array}$} & \multirow{2}{*}{ 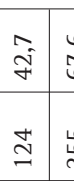 } & \multicolumn{2}{|r|}{$\stackrel{+}{\stackrel{t}{0}}$} & \multicolumn{2}{|r|}{ gे } & \multicolumn{2}{|r|}{$\begin{array}{l}n \\
\hat{n}^{2}\end{array}$} \\
\hline & & & & & & & & & & & & & & 岕 & 缉 & & & & $\infty$ \\
\hline \multirow{6}{*}{ 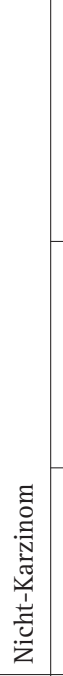 } & \multirow{4}{*}{ 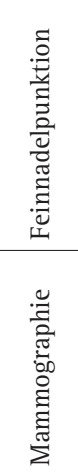 } & 屈。 & \multirow{3}{*}{ 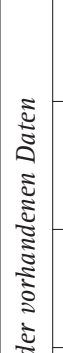 } & $\stackrel{\circ}{i}$ & $\therefore$ & & $\hat{\sigma}^{2}$ & $\begin{array}{ll}m \\
i \\
i\end{array}$ & $\stackrel{m}{F}$ & $\hat{o}$ & $\tilde{n}^{2}$ & के & 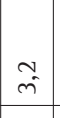 & $\begin{array}{l}+ \\
\pm\end{array}$ & $\hat{\mathrm{m}}$ & जे ले & $\stackrel{0}{-}$ & $\begin{array}{ll}* \\
m \\
m & \infty \\
-1\end{array}$ & $\begin{array}{l}\tilde{L} \\
\sigma^{2}\end{array}$ \\
\hline & & z & & రి & $\overrightarrow{0}$ & & ฟै & $\underset{m}{\mathscr{m}} \approx$ & : & $\hat{m}$ & $\stackrel{\hat{2}}{=}$ & $\frac{m}{m}$ & $\stackrel{\Xi}{\Xi}$ & 占 & 㶽 & जै & & $\stackrel{\infty}{\infty} \tilde{n}$ & $\infty$ \\
\hline & & 応。 & & $\begin{array}{l}0 \\
\infty^{\circ} \\
\end{array}$ & $\begin{array}{l}0 \\
\stackrel{i}{i}\end{array}$ & & $\begin{array}{l}\vec{d} \\
\vec{d}\end{array}$ & $\begin{array}{l}\forall \tilde{v} \\
\vec{\sim} \\
\tilde{v}\end{array}$ & $\therefore$ & $\stackrel{2}{2}$ & $\hat{\sigma}$ & $\stackrel{\infty}{+}$ & $\stackrel{+}{\stackrel{+}{\sim}}$ & $\stackrel{\substack{\infty \\
\infty}}{n}$ & $\stackrel{+}{\circ}$ & $\begin{array}{l}0 \\
\dot{i} \\
i\end{array}$ & $\stackrel{\infty}{2}^{2}$ & $\stackrel{+}{+}$ & $\begin{array}{l}0 \\
\infty^{\circ} \\
-\end{array}$ \\
\hline & & z & 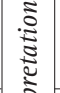 & : & 5 & & ڤิ) & $\stackrel{f}{m} \stackrel{2}{m}$ & $\stackrel{\infty}{\infty}$ & 品 & ț & $\frac{m}{m}$ & $\stackrel{\stackrel{J}{J}}{二}$ & $\stackrel{n}{m}$ & 缉 & $\stackrel{n}{\sigma} \underset{\infty}{\infty}$ & $\tilde{\sigma}$ & ஜ & ळ \\
\hline & \multirow{2}{*}{ 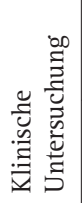 } & \multirow[t]{2}{*}{ 届 } & \multirow{2}{*}{ 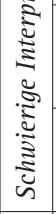 } & 官 & \multicolumn{2}{|l|}{$\begin{array}{l}\stackrel{\circ}{m} \\
\stackrel{m}{m}\end{array}$} & $\begin{array}{l}\infty \\
\infty \\
\sim \\
\sim \\
\sim\end{array}$ & iे & \multicolumn{2}{|c|}{$\begin{array}{ll}0 \\
\circ\end{array}$} & $\vec{i}$ & $\overline{\hat{i}}$ & $\begin{array}{c}m \\
\hat{q} \\
\hat{q}\end{array}$ & $\hat{\text { iे }}$ & $\begin{array}{l}0 \\
\pm \\
\pm\end{array}$ & 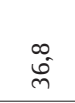 & $\vec{i}$ & $\stackrel{m}{=}$ & $\overline{0}$ \\
\hline & & & & 。ㅇㅇ & $\overrightarrow{6}$ & & $\underset{\sim}{\stackrel{D}{~}}$ & ్ㅠ & $\stackrel{m}{\Xi}$ & 品 & 苂 & $\frac{m}{m}$ & $\stackrel{\Xi}{I}$ & 怘 & 占 & $\stackrel{n}{\sigma}$ & ฮ & $\hat{n}$ & $\infty$ \\
\hline & $\stackrel{\breve{s}}{\stackrel{\Delta}{\leftrightarrow}}$ & वे : & 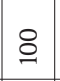 & $\begin{array}{l}\infty^{\circ} \\
\sigma^{\circ}\end{array}$ & 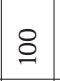 & के & ڤิ & 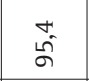 & & ڤ్ & 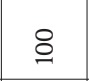 & 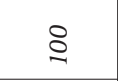 & 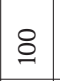 & हे & बे & ผี & 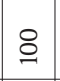 & 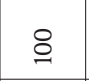 & $\stackrel{+}{\infty}$ \\
\hline & $\begin{array}{l}\frac{1}{0} \\
\stackrel{0}{\Xi} \\
\end{array}$ & z & $\tilde{m}$ & $\stackrel{t}{n}$ & $\infty$ & 忥焉 & 号 & 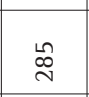 & & $\stackrel{\infty}{\infty}$ & $\stackrel{\infty}{n}$ & 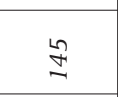 & $\cong$ & I & $\stackrel{M}{\exists}$ & ڤ̆ & in & n. & 8 \\
\hline & 咅 & वे : & 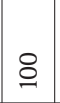 & ڤి & $\begin{array}{l}0 \\
\dot{a}\end{array}$ & $\begin{array}{cc}+\pi \\
\infty \\
\infty \\
\infty\end{array}$ & i̊ & 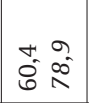 & \begin{tabular}{|c|}
0 \\
$\infty$
\end{tabular} & $\begin{array}{l}\hat{n} \\
\tilde{\theta}\end{array}$ & के & $\hat{a}$ & $\hat{\tilde{j}}$ & : & సે & 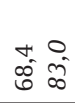 & fं & 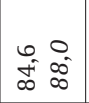 & $\hat{\text { nे }}$ \\
\hline & 穿 & z & $m$ & $\frac{\pi}{n}$ & $\approx$ & 点蛋 & 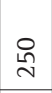 & $\stackrel{\stackrel{\infty}{\infty} \underset{\sim}{\sim} \stackrel{\infty}{\sim}}{\sim}$ & $\bar{m}$ & in & $\stackrel{\infty}{n}$ & $\stackrel{\mathscr{Z}}{ \pm}$ & $\cong$ & f & $\stackrel{\vartheta}{=}$ & ఇू & in & $\stackrel{\sim}{\sim} \stackrel{\llcorner}{\sim}$ & 6 \\
\hline & $\begin{array}{l}\stackrel{.}{\bar{z}} \\
\text { : } \\
\text { : }\end{array}$ & $\vec{x} \div$ & \begin{tabular}{|l|}
$\infty$ \\
+ \\
$\infty$
\end{tabular} & $\begin{array}{l}0 \\
\dot{\infty} \\
\infty\end{array}$ & $\begin{array}{l}0 \\
\dot{f} \\
0\end{array}$ & $\begin{array}{l}\infty \\
\text { w. } \\
\infty \\
\infty \\
\infty \\
\infty\end{array}$ & 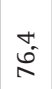 & 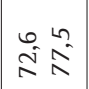 & $\hat{i}$ & gે & $\hat{D}$ & $\begin{array}{c}0 \\
\text { aे }\end{array}$ & $\overrightarrow{0}$ & $\begin{array}{l}\text { s. } \\
\text { á }\end{array}$ & $\begin{array}{l}\infty \\
\stackrel{\infty}{\infty} \\
\end{array}$ & $\vec{\Sigma}$ & $\begin{array}{c}\hat{\infty} \\
\bar{\infty}\end{array}$ & ڤू & $\stackrel{H}{\circ}$ \\
\hline & 彥 & z & $\tilde{m}$ & $\stackrel{\Delta}{E}$ & $\approx$ & 氙焉 & 号 & 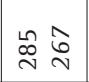 & ב & $\stackrel{t}{O}$ & $\stackrel{\infty}{\curvearrowleft}$ & $\stackrel{L}{ \pm}$ & 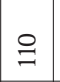 & I & $\stackrel{\varrho}{=}$ & જิ શે & in & i & $\tilde{6}$ \\
\hline & 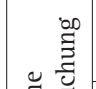 & $\stackrel{2}{\approx} \therefore$ & $\begin{array}{l}\circ \\
\hat{a}\end{array}$ & o & $\begin{array}{l}0 \\
\dot{\delta} \\
0\end{array}$ & $\begin{array}{l}06 \\
20 \\
20\end{array}$ & $\begin{array}{l}0 \\
2\end{array}$ & $\underset{\infty}{\infty}$ & $\begin{array}{l}0 \\
\infty \\
\infty\end{array}$ & $\vec{\infty}$ & \begin{tabular}{l}
$\infty$ \\
\multirow{\sigma}{\sigma}{}
\end{tabular} & $\begin{array}{l}0 \\
\infty \\
\infty\end{array}$ & $\overrightarrow{0}$ & ळे & $\begin{array}{l}\text { o } \\
+\infty\end{array}$ & $\frac{\partial}{\infty}$ & $\begin{array}{l}\hat{\sigma} \\
\dot{b} \\
\curvearrowleft\end{array}$ & $\bar{\infty}$ & $\underset{\substack{a \\
\infty}}{a}$ \\
\hline $\begin{array}{l}\text { त्ञ } \\
\text { त्ञ } \\
\end{array}$ & 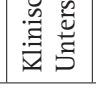 & $z$ & $\tilde{m}$ & $\ddot{n}$ & $\approx$ & 茼急 & 怘 & $\stackrel{\stackrel{\leftrightarrow}{\infty}}{\sim}$ & Z & $\stackrel{\Delta}{\triangle}$ & $\stackrel{\infty}{n}$ & \pm & $\cong$ & I & $\stackrel{M}{\exists}$ & $\stackrel{n}{i}$ & in & ते & 0 \\
\hline & 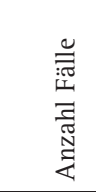 & & 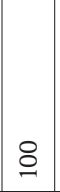 & $\stackrel{\infty}{=}$ & $\cong$ & 芯 & 节 & $\overline{\tilde{\vartheta}}$ & 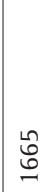 & 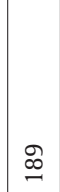 & 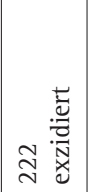 & 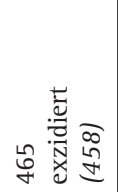 & $\stackrel{\tilde{N}}{\tilde{N}}$ & రิ & | & $\stackrel{\infty}{\Omega}$ & $\underset{\mathrm{N}}{\mathrm{q}}$ & 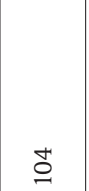 & 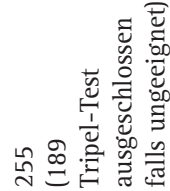 \\
\hline & 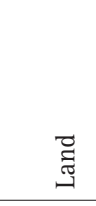 & & $\overleftrightarrow{\widetilde{b}}$ & 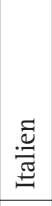 & $\overleftrightarrow{\circlearrowleft}$ & 胥 & 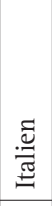 & 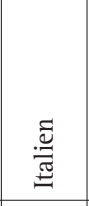 & 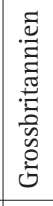 & 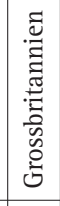 & 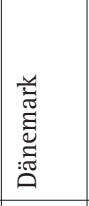 & 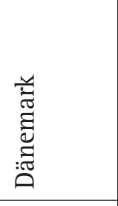 & 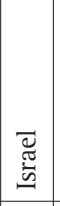 & 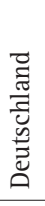 & 爻 & 䔍 & 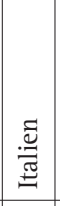 & 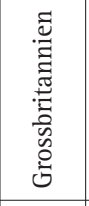 & 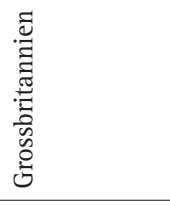 \\
\hline & 㞼 & & $\begin{array}{l}\tilde{\sigma} \\
\text { g } \\
\end{array}$ & $\begin{array}{l}\infty \\
\stackrel{\infty}{\Xi} \\
\end{array}$ & ○ & ڤે & $\begin{array}{l}\stackrel{L}{\infty} \\
\stackrel{2}{\Xi}\end{array}$ & 急 & 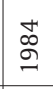 & 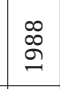 & \begin{tabular}{l}
+ \\
\multirow{2}{*}{} \\
$ٌ$
\end{tabular} & $\begin{array}{l}\infty \\
\stackrel{\infty}{0} \\
.\end{array}$ & 菏 & $\begin{array}{l}0 \\
\stackrel{0}{g}\end{array}$ & $\begin{array}{l}\stackrel{9}{ } \\
\stackrel{2}{9} \\
\end{array}$ & Д & $\begin{array}{l}+ \\
\text { Oे } \\
\sigma\end{array}$ & 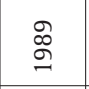 & $\stackrel{\infty}{\stackrel{2}{\sigma}}$ \\
\hline & $\stackrel{\vec{s}}{\vec{z}}$ & & g & $\overrightarrow{0}$ & $\frac{\vec{G}}{\vec{F}}$ & 气્ & 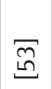 & 苞 & 氙 & 总 & $\sqrt{\tilde{\omega}}$ & 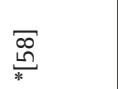 & $\mid$\begin{tabular}{l}
$\vec{\sigma}$ \\
\multirow{2}{*}{} \\
$*$
\end{tabular} & 兽 & $\frac{\bar{\sigma}}{*}$ & $\widetilde{\widetilde{G}}$ & 产 & 疋 & 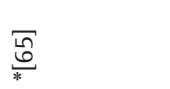 \\
\hline
\end{tabular}

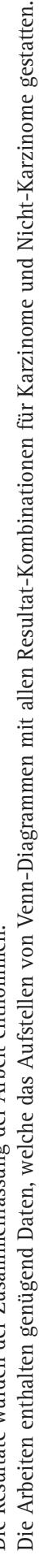




\section{Literatur}

1 Clinical practice guidelines for the care and treatment of breast cancer. A Canadian consensus document. Can Med Assoc J 1998;153(3 Suppl.):S3-S8.

2 Bundesamt für Statistik (Hrsg.). Statistisches Jahrbuch der Schweiz 2000. Zürich: NZZ; 1999.

3 Irwig L, Macashill P. Evidence relevant to guidelines for the investigation of breast symptoms. NHMRC National Breast Cancer Center 1997. ISBN 1876319046. http://www.nbcc.org.au/pages/info/resource/nbccpubs/ brcasymp/sec4.htm\#

4 Page DL. The clinical significance of atypical hyperplasia, multicentricity and bilaterality. In: Wise L, Johnson M Jr (editors). Breast cancer. Controversies in management. New York: Futura Publishing; 1994. p. 203-216.

5 Donegan WL, Spratt JS (editors). Cancer of the breast. Philadelphia: W.B. Saunders; 1995. p. 398.

6 Frykberg E, Bland KI. Overview of the biology and management of ductal carcinoma in situ. Cancer 1994;74:350-62.

7 Kurtz JM, Amalric R, Brandone H, Ayme Y, Jacquemier J, Pietra JC, et al. Local recurrence after breast conserving surgery and radiotherapy; frequency, time course, and prognosis. Cancer 1989;63:1912-7.

8 Friedrichs K. Genetische Aspekte des Mammakarzinoms. Gynäkologie 1994;27:7-11.

9 Makoto A, Koeffler HP. Li-Frauemeni syndrome and the role of the $\mathrm{p} 53$ tumor suppressor gene in cancer susceptibility. Clin Obst Gyn 1998;41:172-99.

10 Frauser BCJM. Molecular genetics of common gynecological cancers. Molecular Biology in reproductive medicine. Parthenon Publishing; 1999.

11 Berchuk A, et al. Familial breast-ovarian cancer syndromes: BRCA1 and BRCA2. Clin Obstet Gyn 1998;41:157-66.

12 Sandles LG. Familial endometrial adenocarcinoma. Clin Obstet Gyn 1998;41:167-71.

13 Bhatia S, Robison LL, Oberlin 0, Greenberg M, Bunin, Fossati-Bellani F, et al. Breast cancer and other second neoplasms after childhood Hodgkin's disease. N Engl J Med 1996;334:745-51.

14 Colditz GA, Willett WC, Hunter DJ, Stampfer MJ, Manson JE, Hennekens $\mathrm{CH}$, et al. Family history, age, and risk of breast cancer. Prospective data from the Nurses Health Study. JAMA 1993;270:338-43.

15 Canadian cancer statistics, 1996. Toronto: National Cancer Institute of Canada; 1996.

16 Dixon JM, McDonald C, Elton RA, Miller WR. Risk of breast cancer in women with palpable breast cysts: a prospective study. Edinburgh Breast Group. Lancet 1999;353:1742-5.

17 Osuch JR. Abnormalities on physical examination. In: Harris JP, Lippman ME, Morrow M, Hellman S, editors. Diseases of the breast. Philadelphia: Lippincott-Raven; 1996. p. 110-3.

18 Donegan WL. Diagnosis. In: Donegan WL, Spratt JS, editors. Cancer of the breast. Philadelphia: W.B. Saunders; 1995. p. 157-205.

19 Benz J, Köchli OR. Mammakarzinom. In: Gynäkologische Onkologie. 2. Aufl. Berlin: Springer; 1999. S. 9.

20 Paget J. On the disease of the mammary areola preceding cancer of the mammary gland. St. Barth Hosp Rep 1874; 10:87-9.

21 Preece PE, Baum M, Mansel RE, Webster DJ, Fortt RW, Gravelle ICH et al. Importance of mastalgia in operable breast cancer. Br Med J 1982;284:1299-300.

22 Hall DC, Goldstein MK, Stein GH. Progress in manual breast examination. Cancer 1977;40:364-70.

23 Rimsten A, Stenkvist B, Johanson H, Lindgren A. The diagnostic accuracy of palpation and fine needle biopsy and an evaluation of their combined use in the diagnosis of breast lesions: report of a prospective study in 1244 women with symptoms. Ann Surg 1975;182:1-8.

24 van Dam PA, Van Goethem MLA, Kersschot E, Vervliet J, Van den Veyver IB, De Schepper A, et al. Palpable solid breast masses: retrospective single- and multimodality evaluations of 201 lesions. Radiology 1988;166:435-9.
25 De Wolf CJM, Perry NM. European guidelines for quality assurance in mammography screening. 2nd edition Luxembourg: Office of official publication of the European Communities; 1996.

26 Sickles EA, Klein DL, Goodson WH, Hunt TK. Mammography after needle aspiration of palpable breast masses. Am J Surg 1983;145:395-7.

27 Kopans DB, Meyer JE, Lindfors KK. Whole-breast US imaging. Four-year follow-up. Radiology 1985;157:505-7.

28 Baker JA, Kornguth PJ, Soo MS, Walsh R, Mengoni P. Sonography of solid breast lesions: Observer variability of lesion description and assessment. AJR 1999;172:1621-5.

29 Ciatto S, Rosselli del Turco M, Catarzi S, Morrone D. The contribution of ultrasonography to the differential diagnosis of breast cancer. Neoplasma 1994;41:341-5.

30 Mödder U, Mosny DS. Rationelle radiologische Diagnostik des Mammakarzinoms. Praxis 1998;87:499-503.

31 Zonderland HM, Hermans J, van de Vijver MJ, Postema S, Tollenaar AEM, van de Velde DJH. Triple diagnostic approach versus ultrasound-guided 18 gauge core biopsy in suspicious breast masses. Breast 1998;7:168-72.

32 Foster RS. Biopsy techniques. In: Harris JP, Lippman ME, Morrow M, Hellman S, editors. Diseases of the breast. Philadelphia: Lippincott-Raven; 1996. p. 133-8.

33 Kline TS. Survey of aspiration biopsy cytology of the breast. Diagn Cytopathol 1991;7:98-105.

34 Layfield LJ, Glasgow BJ, Cramer H. Fine-needle aspiration in the management of breast masses. Pathol Annu 1989; 24:23-62.

35 Willis SL, Ramzy I. Analysis of false results in a series of 835 fine needle aspirates of breast lesions. Acta Cytol 1995;39:858-64.

36 Ballo MS, Sneige N. Can core needle biopsy replace fine-needle aspiration cytology in the diagnosis of palpable breast carcinoma? A comparative study of 124 women. Cancer 1996;78:773-7.

37 Donegan WL. Evaluation of a palpable breast mass. N Engl J Med 1992;327:937-42.

38 Minkowitz S, Moskowitz R, Khafil RA, Alderete MN. TRU-CUT needle biopsy of the breast. Cancer 1986;57:320-3.

39 Donegan WL. Staging and primary treatment. In: Donegan WL, Spratt JS, editors. Cancer of the breast. Philadelphia: W.B. Saunders; 1995. p. 357-442.

40 Caines JS, McPhee MD, Konok GP, Wright BA. Stereotaxis needle core biopsy of breast lesions using a regular mammography table with an adaptable stereotaxis device. AJR Am J Roentgenol 1994;163:317-21.

41 Caines JS, Chantziantoniou K, Wright BA, Konok GP, Iles S, Bodurtha A, et al. Nova Scotia Breast Screening Program experience: use of needle core biopsy in the diagnosis of screening-detected abnormalities. Radiology 1996; 198:125-30

42 Lawson R. Implications of surface temperatures in the diagnosis of breast cancer. CMAJ 1956;75:309-10.

43 Moskowitz M. Breast imaging. In: Donegan WL, Spratt JS, editors. Cancer of the breast. Philadelphia: W.B. Saunders; 1995. p. 206-39.

44 Report of the Working Group to Review NCI/ACS Breast Cancer Detection Demonstration Projects. Bethesda (MD) National Cancer Institute; 1977.

45 Threatt B, Norbeck JM, Ullman NS, Roselle P. Thermography and breast cancer: an analysis of blind reading. Ann N Y Acad Sci 1980;335:501-19.

46 Lerman C, Daly M, Walsh WP, Resch N, Seay J, Barsevick A, et al. Communication between patients with breast cancer and health care providers. Cancer 1993;72:2612-20.

47 Roberts CS, Cox CE, Reintgen DS, Baile WF, Gibertini MG. Influence of physician communication on newly diagnosed breast patients' psychologic adjustment and decision-making. Cancer 1994;74:336-41.

48 Ciatto S, Bravetti P, Cariaggi P. Significance of nipple discharge clinical patterns in the selection of cases for cytologic examination. Acta Cytologica 1986;30:17-20. 
49 Atamdede F, Isaacs J. The role of fine-needle aspiration in the diagnosis of breast lesions. Genealogic Oncology 1993;50:159-63.

50 Assarelli A, Guzzon A, Pilotti S, Quagliuolo V, Bono A, Di Pietro S. Accuracy of breast cancer diagnosis by physical, radiologic and cytologic combined examinations. Tumori 1983;69:137-41.

51 Butler J, Vargas H, Worthen N, Wilson S. Accuracy of combined clinical-mammographic-cytologic diagnosis of dominant breast masses. A prospective study. Arch Surg 1990;125:893-5.

52 Ciatto S, Cariaggi P, Bulgaresi P, Confortini M, Bonardi R. Fine-needle aspiration cytology of the breast: review of 9,533 consecutive cases. The Breast 1993;2:87-90.

53 Di Pietro S, Fariselli G, Bandieramonte G, Coopmans de Yoldi G, Guzzon A, Viganotti G, Pilotti S. Systematic use of the clinical-mammographic cytology triplet for the early diagnosis of mammary carcinoma. Tumori 1985;71:179-85.

54 Di Pietro S, Fariselli G, Bandieramonte G, Lepera P, Coopmans de Yoldi G, Viganotti G, Pilotti S. Diagnostic efficacy of the clinical-radiological-cytological triad in solid breast lumps: results of a second prospective study on 631 patients. Eur J Surg Oncol 1987;13:335-40.

55 Dixon J, Anderson T, Lamb J, Nixon S, Forrest A. Fine-needle aspiration cytology, in relationships to clinical examination and mammography in the diagnosis of a solid breast mass. Br J Surg 1984;81:593-6.

56 Hansell D, Cooke J, Parsons C. The accuracy of mammography alone and in combination with clinical examination and cytology in the detection of breast cancer. Clin Radiol 1988;39:150-3.

57 Hermansen C, Poulsen H, Jensen J, Langfeldt B, Steenskov V, Frederiksen P, Jensen 0. Palpable breast tumours: 'triple-diagnosis' and operative strategy. Acta Chir Scand 1984;150:625-8.
58 Hermansen C, Skovgaard H, Jensen J, Langfeldt B, Steenskov V, Frederiksen P, Jensen 0. Diagnostic reliability of combined physical examination, mammography and fine-needle puncture ('triple test') in breast tumours. Cancer 1987;60:1866-71.

59 Kaufman Z, Shpitz B, Shapiro M, Rona R, Lew S, Dinbar A. Triple approach in the diagnosis of dominant breast masses: combined physical examination, mammography and fineneedle aspiration. Journal of Surgical Oncology 1994; 56:254-7.

60 Kreuzer G, Boquoi E. Aspiration biopsy cytology, mammography and clinical exploration: a modern set-up in diagnosis of tumours of the breast. Acta Cytologica 1976; 20:319-23.

61 Layfield L, Glasgow B, Cramer H. Fine-needle aspiration in the management of breast masses. Pathology Annual 1989;2:23-62.

62 Martelli G, Pilotti S, Coopmans de Yoldi G, Viganoti G, Fariselli G, Lepera P, Moglia D. Diagnostic efficacy of physical examination, mammography, fine-needle aspiration cytology (triple test) in solid breast lumps: an analysis of 1,708 consecutive cases. Tumori 1990;76:476-9.

63 Negri S, Bonetti F, Capitanio A, Bonzanini M. Preoperative diagnostic accuracy of fine-needle aspiration in the management of breast lesions: comparison of specificity and sensitivity with clinical examination mammography, echography and thermography in 249 patients. Diagnostic Cytopathol 1994;11:4-8.

64 Spalding T, Dewar E, Buchanan J, Mugridge A, Conroy B. Fine-needle aspiration cytology as part of a three-stage diagnostic approach to breast lumps in a district general hospital. Journal of the Royal Naval Medical Service 1989; 75:5-9.

65 Thomas JM, Fitzharris B, Redding W, Williams J, Trott P, Powles $\mathrm{T}$, et al. Clinical examination, xeromammography and fine-needle aspiration cytology in diagnosis of breast tumours. Br Med J 1978;2:1139-41. 